\title{
Room Temperature Direct Band Gap Emission from Ge p-i-n Heterojunction Photodiodes
}

\author{
E. Kasper, ${ }^{1}$ M. Oehme, ${ }^{1}$ T. Arguirov, ${ }^{2,3}{ }^{3}$. Werner, ${ }^{1}$ M. Kittler, ${ }^{2,3}$ and J. Schulze ${ }^{1}$ \\ ${ }^{1}$ Institut für Halbleitertechnik (IHT), University of Stuttgart, 70659 Stuttgart, Germany \\ ${ }^{2}$ Leibniz-Institut für Innovative Mikroelektronik (IHP), 15236 Frankfurt (Oder), Germany \\ ${ }^{3}$ Joint Lab IHP/BTU Cottbus, 03013 Cottbus, Germany
}

Correspondence should be addressed to M. Oehme, oehme@iht.uni-stuttgart.de

Received 18 October 2011; Accepted 12 December 2011

Academic Editor: Ram Gupta

Copyright (C) 2012 E. Kasper et al. This is an open access article distributed under the Creative Commons Attribution License, which permits unrestricted use, distribution, and reproduction in any medium, provided the original work is properly cited.

\begin{abstract}
Room temperature direct band gap emission is observed for Si-substrate-based Ge p-i-n heterojunction photodiode structures operated under forward bias. Comparisons of electroluminescence with photoluminescence spectra allow separating emission from intrinsic $\mathrm{Ge}(0.8 \mathrm{eV})$ and highly doped $\mathrm{Ge}(0.73 \mathrm{eV})$. Electroluminescence stems from carrier injection into the intrinsic layer, whereas photoluminescence originates from the highly $\mathrm{n}$-doped top layer because the exciting visible laser wavelength is strongly absorbed in Ge. High doping levels led to an apparent band gap narrowing from carrier-impurity interaction. The emission shifts to higher wavelengths with increasing current level which is explained by device heating. The heterostructure layer sequence and the light emitting device are similar to earlier presented photodetectors. This is an important aspect for monolithic integration of silicon microelectronics and silicon photonics.
\end{abstract}

\section{Introduction}

Progress in Si-based photonics from Ge/Si heterostructures attracts worldwide attention [1]. Photonics and optoelectronics play an essential role in many areas of applications [2] as in telecommunication, information technology, and optical interconnect systems. Integration of Si-based microelectronics and optoelectronic devices would be greatly enhanced if similar facilities and technologies can be used. One approach is the development of optoelectronic components based on Si compatible materials.

Waveguiding in silicon-on-insulator (SOI) structures, high speed detecting by Ge/Si heterostructure devices [3, 4], and signal modulation by interferometric principles were demonstrated in the last decades. In the last years, small area absorption modulators based on electric field modification of material properties (quantum-confined Stark effect, Franz-Keldysh effect) were developed [5]. However, the realization of light emitters with a Si base is still challenging. Due to the fact that it is an indirect semiconductor with a relatively large direct band gap $(3.2 \mathrm{eV})$, Si itself is not suited for the development of light emitters. An alternative and compatible material is Ge. Ge is also an indirect semiconductor but the direct band gap of $0.8 \mathrm{eV}$ is only slightly larger than the indirect one $(\Delta E=136 \mathrm{meV})$. Furthermore, $0.8 \mathrm{eV}$ corresponds to the required communication wavelength of $1,550 \mathrm{~nm}[6]$. The small energy difference between the direct and the indirect band gap should open the possibility for light emitting devices with reasonable quantum efficiencies.

The direct gap photoluminescence of $\mathrm{Ge}$ at a temperature of $2 \mathrm{~K}$ was measured already in 1978 [7]. Tensile strain and high n-doping increase the luminescence emission efficiency [8]. In the last time demonstrations of direct band gap luminescence and optical gain on $\mathrm{Ge} / \mathrm{Si}$ diodes were reported [9-12]. In this paper we demonstrate an integration friendly Ge p-i-n heterojunction photodiode, which is used for both, lightdetection and light emitting, by only changing the bias from reverse to forward.

\section{Diode Structure and Low Temperature Annealing}

The Ge p-i-n heterojunction photodiode is produced in a quasiplanar technology. B-doped (100) oriented Si substrates 


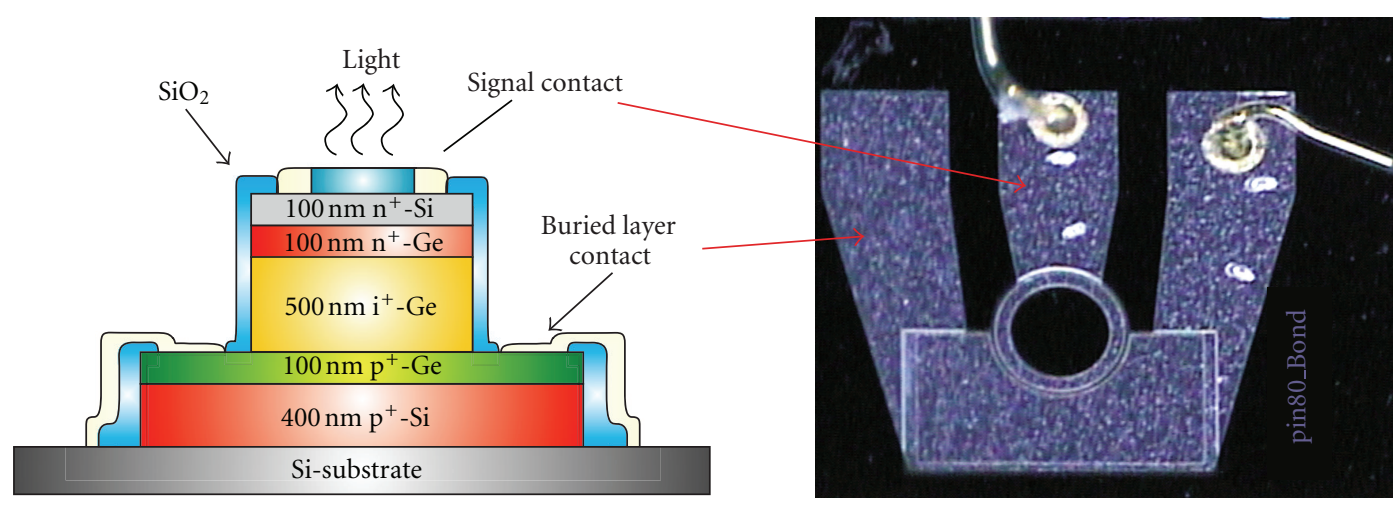

FIGURE 1: Schematic cross-section of a Ge p-i-n heterojunction photodiode (left). The right figure shows a microscopy image of a complete device structure with typically ground signal ground contacts. The mesa diameter of the diode and the diameter of the optical window amount $160 \mu \mathrm{m}$ and $100 \mu \mathrm{m}$, respectively.

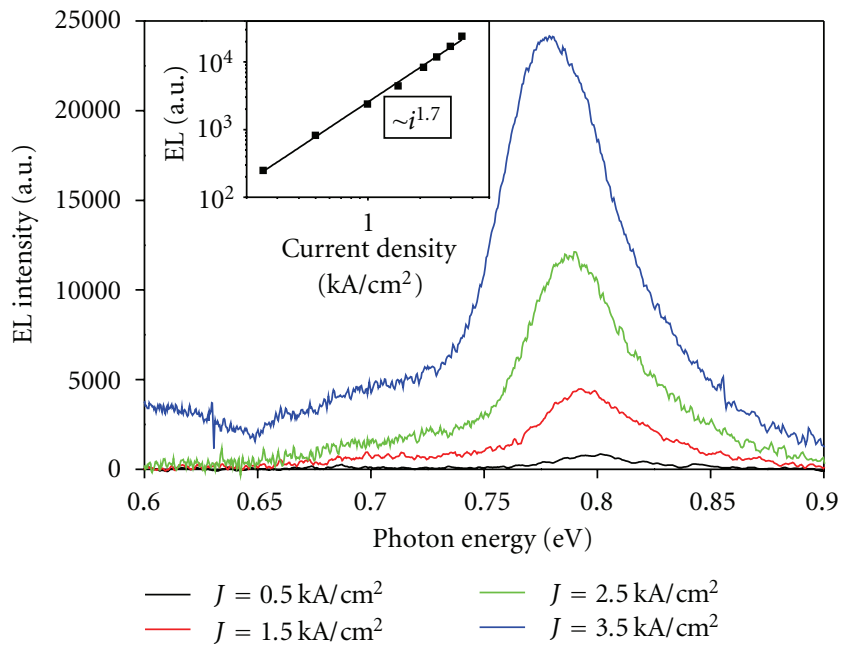

FIGURE 2: Electroluminescence spectrum of the Ge p-i-n heterojunction photodiode as function of the electrical drive current density. The inset shows the intensity of the electroluminescence at peak position in dependence of the current driven trough the device.

with a high specific resistance $\rho>1000 \Omega \mathrm{cm}$ are used. The complete layer sequence is grown with a solid source SiGe MBE (molecular beam epitaxy) equipment for $150 \mathrm{~mm}$ diameter substrates with a base pressure less than $10^{-10} \mathrm{mbar}$ [13]. Based on a buried layer technology, the backside contact is led to the front side. The schematic cross-section of the Ge p-i-n heterojunction photodiode is shown in Figure 1.

The layer growth starts with a $400 \mathrm{~nm}$ thick very high B-doped $\left(10^{20} \mathrm{~cm}^{-3}\right)$ Si-buried layer. For the lattice accommodation between $\mathrm{Ge}$ and $\mathrm{Si}$, a special 3 stage deposition and annealing process was developed. In the 1st stage, a highly B-doped $\left(10^{20} \mathrm{~cm}^{-3}\right)$ strain-relaxed Ge buffer layer (virtual substrate) with a thickness of $50 \mathrm{~nm}$ is deposited at a growth temperature of $330^{\circ} \mathrm{C}$. An annealing step at $850^{\circ} \mathrm{C}$ considerably reduces the original threading dislocation density in the virtual substrate. In the 2nd stage, a second $50 \mathrm{~nm}$ Ge layer (also $10^{20} \mathrm{~cm}^{-3} \mathrm{~B}$-doped) is grown at $330^{\circ} \mathrm{C}$ followed by a $620^{\circ} \mathrm{C}$ annealing step. After forming the $\mathrm{p}^{+}$-type doped buried contact the intrinsic region is also grown at $330^{\circ} \mathrm{C}$ with a thickness of $500 \mathrm{~nm}$ (3rd stage). The growth temperature of $330^{\circ} \mathrm{C}$ is low enough that the surface segregation of $\mathrm{B}$ is negligible and the doping concentration drops more than 4 orders of magnitude within a few nanometers between the buried contact and the intrinsic region. After growth the intrinsic region is also annealed at $700^{\circ} \mathrm{C}$. The $\mathrm{n}^{+}$-doped top contact is finally realized as highly Sb-doped Ge/Si heterojunction contact [14] with a very high $\mathrm{Sb}$ doping concentration in the range greater than $10^{20} \mathrm{~cm}^{-3}$ [15]. The Ge p-i-n heterojunction photodiodes are realized in a double-mesa layout with radii between $1.5 \mu \mathrm{m}$ and $80 \mu \mathrm{m}$. After double mesa etching a $345 \mathrm{~nm}$ thick $\mathrm{SiO}_{2}$ passivation is used to insulate the mesa sidewalls from the signal contacts. Figure 1 shows a microscopy image of the complete device structure with a radius of $80 \mu \mathrm{m}$. The $\mathrm{Al}$ contacts have a ground-signal-ground configuration for easy on-wafer testing with RF-probes and a window opening for optical experiments.

\section{Experimental Results}

As detector the photodiode is reverse biased [16, 17] or favorably zero biased [18]. Under forward bias the electrons and holes are injected from their respective highly doped sides into the intrinsic depletion layer and recombine there. In indirect semiconductors the fastest recombination channel via mid gap recombination levels provided by metals and defects is radiation less which weakens the indirect transition. As mentioned above the lowest direct transition energy in $\mathrm{Ge}$ is only $136 \mathrm{meV}$ above the indirect band gap which delivers a finite occupation probability for direct levels. At room temperature the occupation probability of the direct states is only a fraction of a percent compared to the indirect states (Boltzmann approximation). With increasing quasi-Fermi level at higher current injection the occupation increases significantly when the electron quasi-Fermi level enters the conduction band (Fermi statistics). Already, at rather low direct states occupation probabilities the radiative recombination from these states dominates because of weak competition from indirect states. 


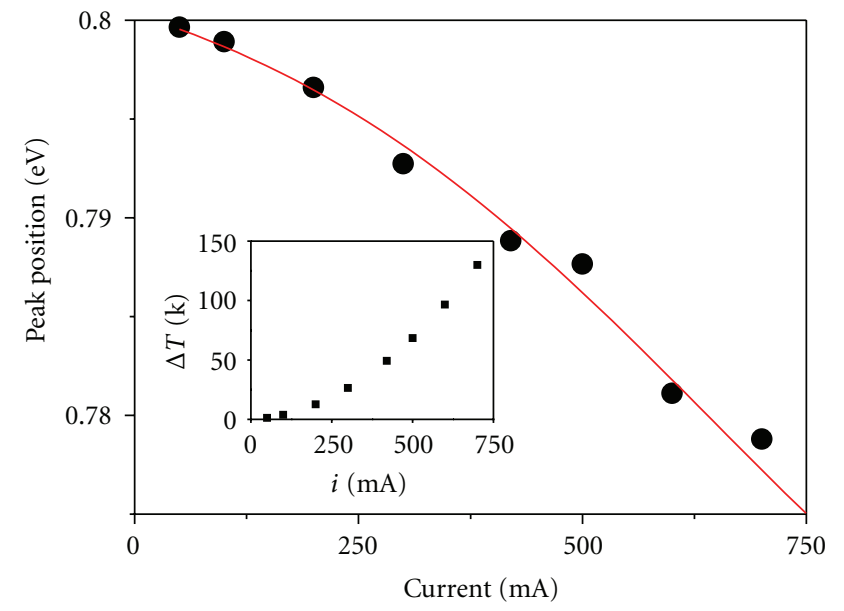

Figure 3: Peak position of EL spectra as function of electrical current. The inset shows the temperature increase $\Delta \mathrm{T}$ by current heating $\left(R_{\mathrm{th}}=40 \mathrm{~K} / \mathrm{W}\right.$ assumed $)$.

We compared the direct bandgap emission with electroluminescence (EL) and photoluminescence (PL) directly on the vertical device structures. For our PL investigations reported below we used a standard spectroscopic setup. The Ge samples were excited by means of a frequencydoubled Nd:YAG diode-pumped solid-state laser, emitting continuous-wave at wavelength of $532 \mathrm{~nm}$. The spot of the laser beam at the surface of the sample was about $100 \mu \mathrm{m}$ in diameter. The laser power used for excitation was in the range from $100 \mathrm{~mW}$ to $1 \mathrm{~W}$. The absorption depth at this wavelength is about $40 \mathrm{~nm}$ within the highly n-doped $\mathrm{Ge}$ top layer. The luminescence was collected by a parabolic mirror and spectrally analyzed by a monochromator $(0.64 \mathrm{~m}$ focal length, F/5.2 aperture) equipped with a $300 \mathrm{l} / \mathrm{mm}$ grating and an InSb detector. The excitation beam was chopped at $133 \mathrm{~Hz}$ and lock-in technique was used to process the signals. For EL measurements we used the same setup, but forward biasing the sample diodes with current pulses for injection of minority carriers. Different excitation levels were achieved by setting different amplitudes of the current pulses. For EL excitation, electrical forward bias was given on $160 \mu \mathrm{m}$ mesa diameter diodes with bonded contacts (see Figure 1, right). Intensity ratio of direct-toindirect transitions, emission wavelength, and temperature and power dependence are considered as significant numbers for direct bandgap emission.

The emission spectrum (see Figure 2) in EL demonstrates clearly the dominance of radiation from direct transitions at around $0.8 \mathrm{eV}$. Indirect transitions at around $0.66 \mathrm{eV}$ are much weaker. The spectrum in Figure 2 is shown down to $0.6 \mathrm{eV}$. We found also a peak around $0.45 \mathrm{eV}$ which we assign to radiative recombination at dislocations. It is weaker than the direct transition and not further considered in this paper.

The current density dependence is superlinear (roughly $\mathrm{I}^{1.7}$, see inset of Figure 2) which is an indication of higher occupation probabilities of direct states when the quasiFermi energies are increased with higher current densities. The emission intensity increases with higher ambient

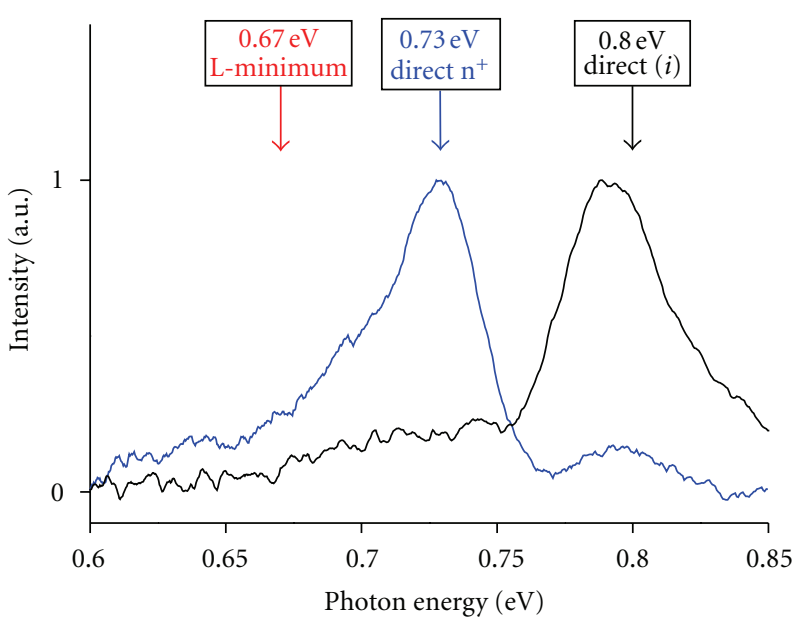

- PL diode

EL diode

Figure 4: Comparison of EL (black) and PL (blue) from the same diode. The energy positions of indirect $(0.67 \mathrm{eV})$ and direct transition from intrinsic $\mathrm{Ge}(0.8 \mathrm{eV})$ and $\mathrm{n}^{+}$-type doped $\mathrm{Ge}$ $(0.73 \mathrm{eV})$ are marked.

temperature (factor 10 from $80 \mathrm{~K}$ to $300 \mathrm{~K}$ ) in qualitative agreement with the occupation statistics of higher direct energy levels. Together, intensity ratio of direct to indirect transitions (more than five), main emission wavelength $(0.80 \mathrm{eV})$, and temperature $(300 \mathrm{~K}$ emission stronger than at $80 \mathrm{~K}$ ) and power dependence (superlinear) are considered as significant proof for direct bandgap emission.

The peak position (see Figure 3) decreases nonlinear with current increase which is explained by the current-induced temperature increase. The nonlinear behavior reflects the nonlinear temperature increase (inset of Figure 3) caused by the power consumption increasing superlinear because of series resistance contributions $R_{S} \cdot I^{2}$ at higher current levels.

A comparison of EL with PL in Figure 4 shows at the position at around $0.8 \mathrm{eV}$ a clear detectable but weaker peak for PL. The main signature in PL stems from the $0.73 \mathrm{eV}$ peak which is assigned to direct transitions from the $\mathrm{n}^{+}$-doped top contact layer. The high doping concentration $\left(>10^{20} \mathrm{~cm}^{-3}\right)$ leads to a band gap narrowing. Under this term carrierimpurity interaction effects from donor potentials, impurity band and many electron interactions are summarized. This is well known and taken into account in Si microelectronic device physics, but, to our knowledge, not treated up to now in SiGe photonics [19]. In $\mathrm{Si}$ a doping level of $10^{20} \mathrm{~cm}^{-3}$ leads to a band gap narrowing of $90 \mathrm{meV}$ [20]). This PL observation explains also the broad shoulder on the low energy side of the $0.8 \mathrm{eV}$ EL peak as overlap of indirect transitions $(0.66 \mathrm{eV})$ with radiative recombination in the high-doped regions around the depletion layer.

\section{Conclusion}

For integration concepts of $\mathrm{Ge}$ on Si-based photonics the use of the same device structure for detectors, modulators, and emitters is of paramount importance. We have earlier 
shown that these Ge p-i-n heterojunction photodiodes are fast detectors (>40 GHz see [4]) which can be exploited for modulators with reverse bias swing at moderate reverse bias (the Franz-Keldysh effect, GFP 2010 [21]) and which can be also used as direct band gap emitters at forward bias.

The observed peak intensities at $0.73 \mathrm{eV}$ to $0.8 \mathrm{eV}$ are explained by direct transitions in the highly doped $\mathrm{n}^{+}$-doped Ge top contact layer (due to band gap narrowing) and in the intrinsic Ge region of the diode, respectively.

\section{Acknowledgments}

The authors thank O. Kirfel, M. Sarlija, M. Kaschel, M. Schmid, and D. Hähnel for assistance with the MBE growth, device fabrication, and the useful discussions. This work was supported by the Deutsche Forschungsgemeinschaft (German Research Foundation).

\section{References}

[1] B. Jalali and S. Fathpour, "Silicon photonics," Journal of Lightwave Technology, vol. 24, no. 12, pp. 4600-4615, 2006.

[2] R. Soref, "Silicon photonics: a review of recent literature," Silicon, vol. 2, no. 1, pp. 1-6, 2010.

[3] R. Cohen, M. M. Morse, G. Sarid, Y. Chetrit, D. Rubin, and M. Paniccia, " $31 \mathrm{GHz}$ Ge n-i-p waveguide photodetectors on Silicon on Insulator substrate," in Proceedings of the 4th IEEE/LEOS International Conference on Group IV Photonics, Tokyo, Japan, 2007.

[4] S. Klinger, M. Berroth, M. Kaschel, M. Oehme, and E. Kasper, "Ge-on-Si p-i-n photodiodes with a 3-dB bandwidth of 49 GHz," IEEE Photonics Technology Letters, vol. 21, no. 13, pp. 920-922, 2009.

[5] Y. H. Kuo, Y. K. Lee, Y. Ge et al., "Strong quantumconfined Stark effect in germanium quantum-well structures on silicon," Nature, vol. 437, no. 7063, pp. 1334-1336, 2005.

[6] C. Claeys and E. Simoen, Germanium Based Technologies: From Materials to Devices, Elsevier, Amsterdam, The Netherlands, 2007.

[7] W. Klingenstein and H. Schweizer, "Direct gap recombination in germanium at high excitation level and low temperature," Solid State Electronics, vol. 21, no. 11-12, pp. 1371-1374, 1978.

[8] J. Liu, R. Camacho-Aguilera, J. T. Bessette et al., "Ge-on-Si optoelectronics," Thin Solid Films, vol. 520, no. 8, pp. 3354 3360, 2012.

[9] X. Sun, J. Liu, L. C. Kimerling, and J. Michel, "Roomtemperature direct bandgap electroluminesence from Ge-onSi light-emitting diodes," Optics Letters, vol. 34, no. 8, pp. 1198-1200, 2009.

[10] S. L. Cheng, J. Lu, G. Shambat et al., "Room temperature $1.6 \mu \mathrm{m}$ electroluminescence from Ge light emitting diode on Si substrate," Optics Express, vol. 17, no. 12, pp. 10019-10024, 2009.

[11] J. Liu, X. Sun, L. C. Kimerling, and J. Michel, "Direct-gap optical gain of Ge on Si at room temperature," Optics Letters, vol. 34, no. 11, pp. 1738-1740, 2009.

[12] J. Liu, X. Sun, R. Camacho-Aguilera, L. C. Kimerling, and J. Michel, "Ge-on-Si laser operating at room temperature," Optics Letters, vol. 35, no. 5, pp. 679-681, 2010.

[13] M. Oehme, J. Werner, M. Kaschel, O. Kirfel, and E. Kasper, "Germanium waveguide photodetectors integrated on silicon with MBE," Thin Solid Films, vol. 517, no. 1, pp. 137-139, 2008.

[14] M. Oehme, M. Kaschel, J. Werner et al., "Germanium on silicon photodetectors with broad spectral range," Journal of the Electrochemical Society, vol. 157, no. 2, pp. H144-H148, 2010.

[15] M. Oehme, J. Werner, and E. Kasper, "Molecular beam epitaxy of highly antimony doped germanium on silicon," Journal of Crystal Growth, vol. 310, no. 21, pp. 4531-4534, 2008.

[16] J. Wang and S. Lee, "Ge-photodetectors for Si-based optoelectronic integration," Sensors, vol. 11, no. 1, pp. 696-718, 2011.

[17] M. Oehme, J. Werner, E. Kasper, S. Klinger, and M. Berroth, "Photocurrent analysis of a fast Ge p-i-n detector on Si," Applied Physics Letters, vol. 91, no. 5, Article ID 051108, 3 pages, 2007.

[18] E. Kasper and M. Oehme, "High speed germanium detectors on Si," Physica Status Solidi (C), vol. 5, no. 9, pp. 3144-3149, 2008.

[19] E. Kasper, M. Oehme, T. Aguirov, J. Werner, M. Kittler, and J. Schulze, "Room temperature direct band gap emission from Ge p-i-n heterojunction photodiodes," in Proceedings of the 7th IEEE/LEOS International Conference on Group IV Photonics, Beijing, China, 2010.

[20] D. B. M. Klaassen, J. W. Slotboom, and H. C. de Graaff, "Unified apparent bandgap narrowing in n- and p-type silicon," Solid-State Electronics, vol. 35, no. 2, pp. 125-129, 1992.

[21] M. Schmid, M. Oehme, M. Kaschel, J. Werner, E. Kasper, and J. Schulze, "Franz-Keldysh effect in Germanium on Silicon p-i-n photodetectors," in Proceedings of the 7th IEEE/LEOS International Conference on Group IV Photonics, pp. 329-331, Beijing, China, 2010. 

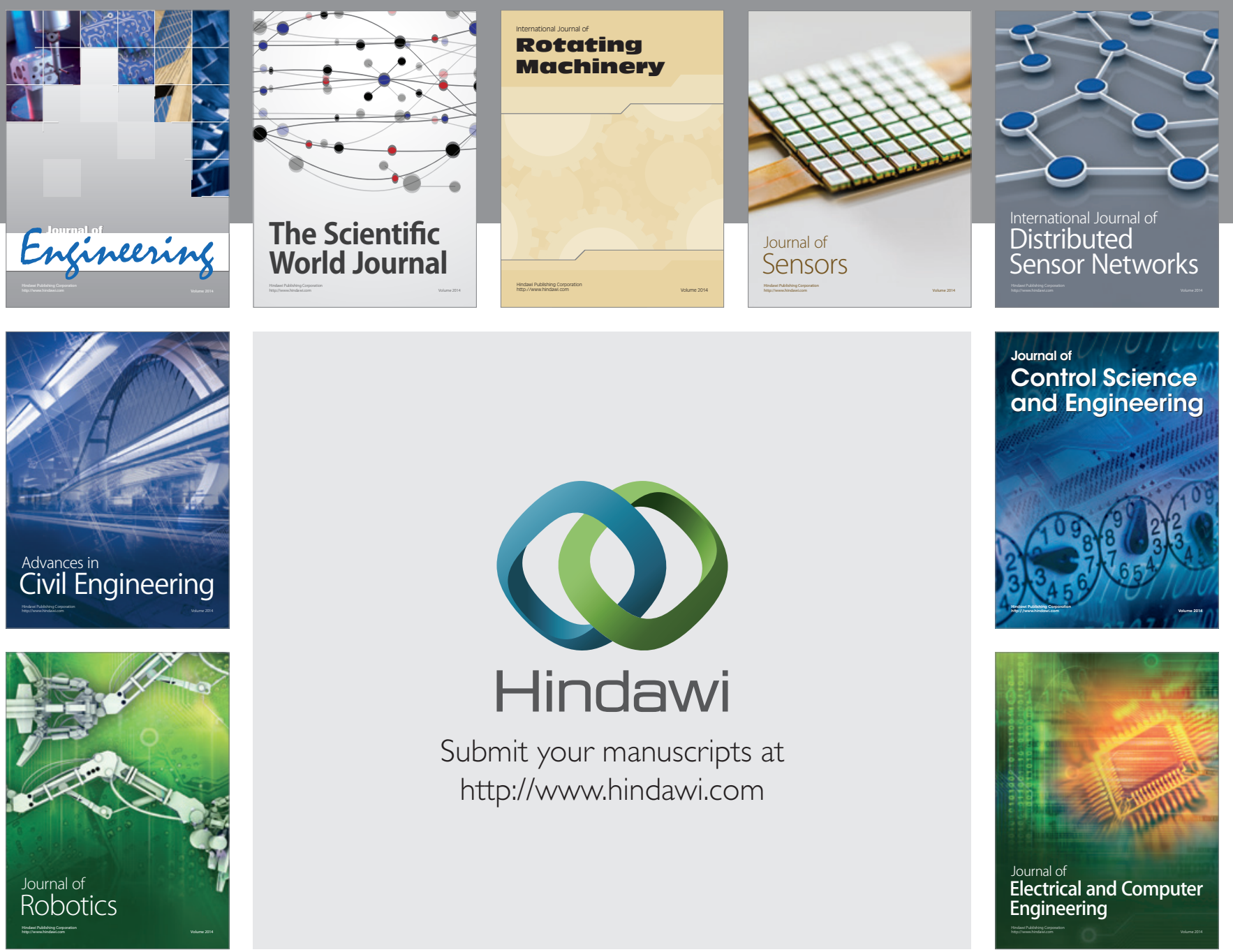

Submit your manuscripts at

http://www.hindawi.com
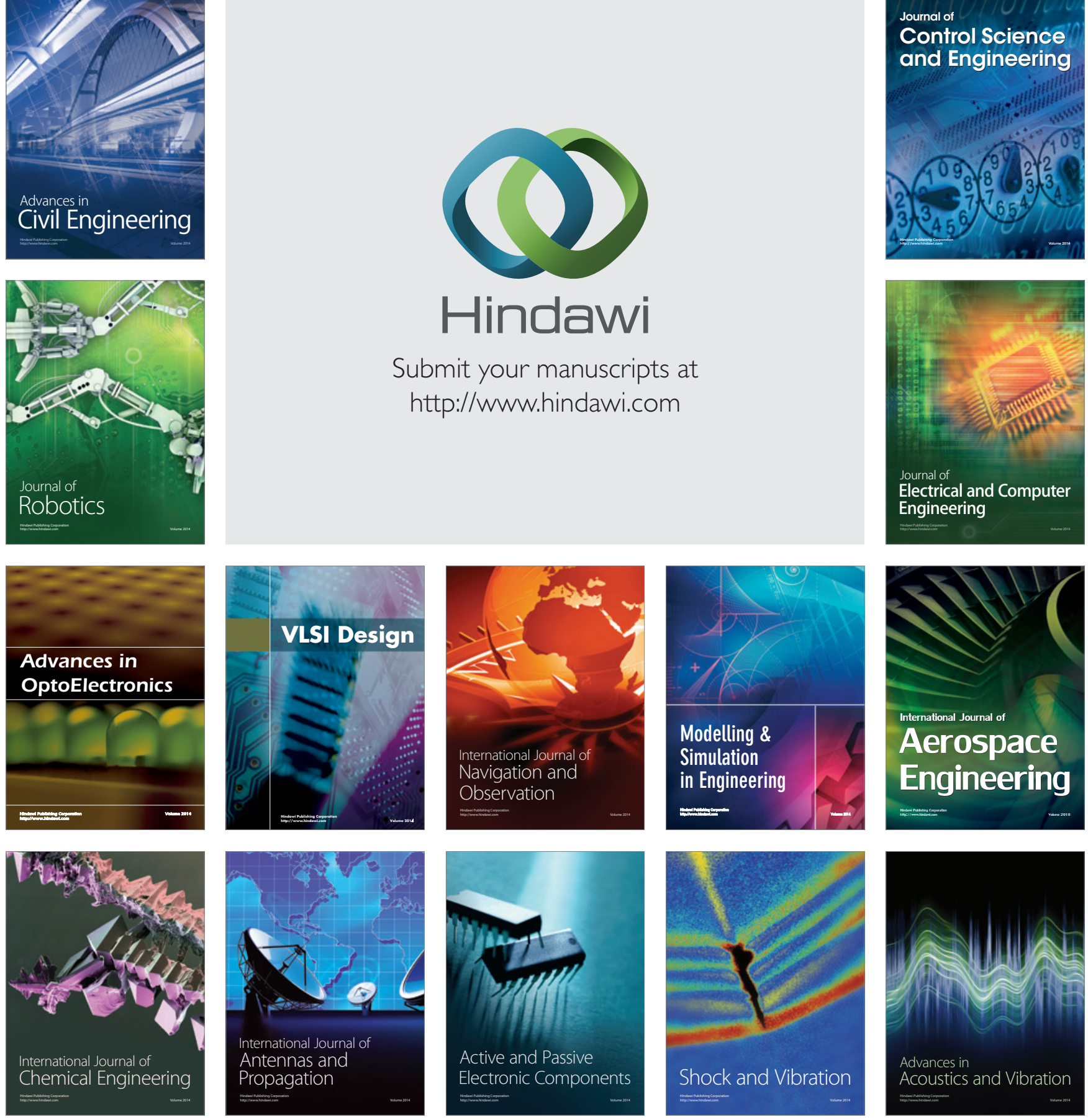